\title{
Association of desaturase activity and C-reactive protein in European children
}

\author{
Maike Wolters' ${ }^{1}$, Claudia Börnhorst' ${ }^{1}$, Heike Schwarz' ${ }^{1}$ Patrizia Risé2 ${ }^{2}$ Claudio Galli², Luis A. Moreno ${ }^{3}$, Valeria Pala ${ }^{4}$, Paola Russo ${ }^{5}$, \\ Toomas Veidebaum 6 , Michael Tornaritis ${ }^{7}$, Arno Fraterman ${ }^{8}$, Stefaan De Henauw ${ }^{9}$, Gabriele Eiben ${ }^{10}$, Lauren Lissner ${ }^{10}$, \\ Dénes Molnár ${ }^{11}$ and Wolfgang Ahrens ${ }^{1,12}$; on behalf of the IDEFICS Consortium.
}

BACKGROUND: Desaturase enzymes influence the fatty acid (FA) composition of body tissues and their activity affects the conversion rate of saturated to monounsaturated FA and of polyunsaturated FA (PUFA) to long-chain PUFA. Desaturase activity has further been shown to be associated with inflammation. We investigate the association between delta-9 (D9D), delta-6 (D6D) and delta-5 desaturase (D5D) activity and highsensitive C-reactive protein (CRP) in young children.

METHODS: In the IDEFICS (Identification and prevention of dietary- and lifestyle-induced health effects in children and infants) cohort study children were examined at baseline (TO) and after 2 y (T1). D9D, D6D, and D5D activities were estimated from T0 product-precursor FA ratios. CRP was measured at T0 and T1. In a subsample of 1,943 children with available information on FA, CRP, and covariates, the cross-sectional and longitudinal associations of desaturase activity and CRP were analyzed.

RESULTS: Cross-sectionally, a D9D increase of 0.01 units was associated with a $11 \%$ higher risk of having a serum CRP $\geq$ Percentile 75 (P75) (OR, 99\% Cl: 1.11 (1.01; 1.22)) whereas D6D and D5D were not associated with CRP. No significant associations were observed between baseline desaturase activity and CRP 2 y later.

CONCLUSION: Cross-sectionally, our results indicate a positive association of D9D and CRP independent of weight status. High D9D activity may increase the risk of subclinical inflammation which is associated with metabolic disorders. As D9D expression increases with higher intake of saturated FA and carbohydrates, dietary changes may influence D9D activity and thus CRP. However, it remains to be investigated whether there is a causal relationship between D9D activity and CRP.

M oderately increased C-reactive protein (CRP) is a marker of subclinical inflammation which is frequently observed in obese subjects and was shown to be predictive for cardiovascular diseases (1). As obesity rates have dramatically increased also in young age groups $(2,3)$, increased CRP is already prevalent in children (1). Inflammatory status is related to the fatty acid (FA) tissue levels and to dietary FA intake $(4,5)$. FA composition of body tissues strongly depends on dietary intake of FA and on the activity of the enzymes involved in the conversion of saturated FA (SFA) to monounsaturated FA (MUFA) and of polyunsaturated FA (PUFA) to long-chain (LC) PUFA, i.e., desaturase and elongase enzymes.

Delta-9 desaturase (D9D, also called stearoyl-CoA desaturase, SCD) catalyzes the desaturation of SFA, preferably palmitic (16:0) and stearic acid (18:0), to the MUFA palmitoleic $(16: 1 \mathrm{n}-7)$ and oleic acid (18:1n-9). It is the rate-limiting enzyme and therefore influences the body composition of these FA. Delta-6 desaturase (D6D) catalyzes the first step in the conversion of the n-6 PUFA linoleic (18:2n-6) and the n-3 PUFA $\alpha$-linolenic acid (18:3n-3) to the LC PUFA arachidonic $(20: 4 n-6)$ and eicosapentaenoic acid (20:5n-3). This step is followed by elongation of the intermediate PUFA and by subsequent desaturation of these intermediates by delta- 5 desaturase (D5D) to the abovementioned LC PUFA. Linoleic acid and $\alpha$-linolenic acid cannot be synthesized and must be supplied by the diet. D6D and D5D are the rate-limiting enzymes for the desaturation steps in the conversion from the essential n- 6 and n-3 precursor PUFA to the LC PUFA products.

According to animal studies, deficiency of D9D prevents fat accumulation and obesity, provides favorable metabolic effects and seems to reduce obesity-associated white adipose tissue inflammation (6). In vitro studies have shown inconsistent results indicating that the effects of D9D on inflammation vary by tissue type $(6,7)$. Low levels of $\mathrm{D} 9 \mathrm{D}$ are associated with decreased levels of cardiovascular risk factors like insulin resistance and dyslipidemia in humans (7-10). Additionally, D9D activity is positively associated with the risk of type 2 diabetes,

\footnotetext{
'Leibniz Institute for Prevention Research and Epidemiology - BIPS, Bremen, Germany; ${ }^{2}$ DiSFeB, Department of Pharmacological and Biomolecular Sciences, University of Milan, Milan, Italy; ${ }^{3}$ Growth, Exercise, Nutrition and Development (GENUD) Research Group, University of Zaragoza, Zaragoza, Spain; ${ }^{4}$ Epidemiology and Prevention Unit, Department of Preventive and Predictive Medicine, Fondazione IRCCS Istituto Nazionale Dei Tumori, Milan, Italy; ${ }^{5}$ Epidemiology and Population Genetics, Institute of Food Sciences, National Research Council, Avellino, Italy; ${ }^{6}$ National Institute for Health Development, Tallinn, Estonia; ${ }^{7}$ Research and Education Institute of Child Health, Strovolos, Cyprus; ${ }^{8}$ Medizinisches Versorgungszentrum Dr. Eberhard und Partner Dortmund, Laboratoriumsmedizin, Dortmund, Germany; ${ }^{9}$ Department of Public Health, Faculty of Medicine and Health Sciences, Ghent University, Ghent, Belgium; ${ }^{10}$ Section for Epidemiology and Social Medicine, Institute of Medicine, Sahlgrenska Academy, University of Gothenburg, Gothenberg, Sweden; ${ }^{11}$ National Institute of Health Promotion, University of Pécs, Pécs, Hungary; ${ }^{12}$ Institute of Statistics, Faculty of Mathematics and Computer Science, University of Bremen, Bremen, Germany. Correspondence: Maike Wolters (wolters@leibniz-bips.de)

Received 3 March 2016; accepted 25 August 2016; advance online publication 19 October 2016. doi:10.1038/pr.2016.186
} 


\section{Articles | wolters et al.}

cardiometabolic diseases $(9,11)$ and with CRP $(12,13)$. D9D expression can be induced by glucose, fructose, SFA, and insulin (7). If the positive association between D9D and CRP observed in adults was based on a causal relationship, it could be hypothesized that changes of dietary patterns may affect CRP levels by modifying D9D activity. This assumption is supported by results of a two-months balanced diet intervention based on nutritional recommendations which decreased both, palmitoleic acid, i.e., the product of estimated D9D activity (calculated as ratio of 16:1n-7 and 16:0), and CRP as well as body weight in metabolically healthy obese adults (14). This may indicate a beneficial effect of reduced D9D activity although no association was observed between FA and D9D with CRP such that other factors like weight loss may have been responsible for the CRP decrease. Palmitoleic acid as an indicator of de novo lipogenesis has been shown to be associated with detrimental metabolic outcomes in several studies (15). In Finnish men, erythrocyte membrane palmitoleic acid was positively associated with CRP (16). In contrast, supplementation of palmitoleic acid for $30 \mathrm{~d}$ reduced CRP levels in a randomized controlled trial with 60 adult subjects (17). However, supplementary intake was higher compared with the intake in the usual Western diet (18).

D6D and D5D conversion products, i.e., the LC PUFA arachidonic and eicosapentaenoic acid influence the fluidity and permeability of cell membranes and serve as precursors for prostaglandins and resolvins with effects on inflammation $(19,20)$. In particular, n-3 LC PUFA exert anti-inflammatory effects by several mechanisms such as inhibiting leukocyte chemotaxis, reducing the production of proinflammatory eicosanoids from n-6 PUFA and of inflammatory cytokines as well as by activating antiinflammatory and inhibiting activation of proinflammatory transcription factors (5).

Single nucleotide polymorphisms of the D5D and D6D encoding FADS genes were shown to be associated with reduced desaturase activity (21) and increased inflammatory markers (22). In Korean adults, estimated D6D was positively associated with CRP $(12,23)$, whereas D5D was negatively related (23). Additionally, estimated D6D was found to be an independent determinant for plasma levels of CRP (23). Also in a case-cohort study of $>1,500$ adults including 400 diabetic subjects, estimated D5D was inversely associated with CRP but no association between D6D and CRP was observed (11).

In a recent study based on a sample of 2 to $<10 \mathrm{y}$ old children, we found that D9D and D6D to be positively associated with BMI and blood triglycerides; and negatively with HDL $\mathrm{Z}$-scores, whereas D5D was negatively associated with BMI and triglycerides in a cross-sectional analysis. When assessing the association between baseline desaturase activities and metabolic outcomes measured 2 y later (follow-up), D9D and D6D were positively associated with the BMI z-score while D6D was inversely associated with HDL (10).

As desaturase activity has been shown to be associated with CRP in adults $(12,23)$, it may also be related to subclinical inflammation in children. Studies investigating the association of desaturase activity and inflammation in children are scarce
(24) and completely missing in younger children. Therefore, this explorative study aims to investigate the cross-sectional and longitudinal associations of desaturase activity with highsensitive CRP in European children aged 2 to $<10 \mathrm{y}$ including a high proportion of overweight and obese children.

\section{RESULTS}

Table 1 shows the characteristics of the cross-sectional and longitudinal analysis sample. The children included in our study had a high prevalence of overweight/obesity (25) because of the oversampling of overweight and obese children (22.6 and $20.5 \%$, respectively). As expected (26), CRP values were slightly higher in girls than in boys and a higher proportion of children with CRP $\geq$ Percentile 75 (P75) were obese $(68.9 \%$ in the cross-sectional population). While D9D and D6D levels were slightly higher with higher CRP values, D5D was slightly lower but only in children in the longitudinal analysis group (Table 1). However, in these descriptive data, effects of covariates are not considered.

Table 2 shows the results of the cross-sectional and longitudinal analyses. Each 1 unit increase of D9D (i.e., increase by 0.01 because of the conversion of the scale) was associated with $11 \%$ higher odds of having a serum CRP level $\geq$ P75 (odds ratio (99\% confidence interval, CI): $1.11(1.01 ; 1.22)$ ) whereas D6D and D5D were not associated with CRP. In the longitudinal analysis, no significant associations between CRP and estimated desaturase activities were detected. Adjustment for baseline CRP z-scores did not alter these results.

No interaction between desaturase activity and sex has been found, i.e., the effects of desaturase activity on CRP did not differ significantly between boys and girls. For this reason results are not reported stratified by sex.

\section{DISCUSSION}

We analyzed cross-sectional and longitudinal associations between estimated desaturase activities and CRP in a large sample of 2 to $<10$ y old European children. Our study confirmed a positive association of D9D with CRP as previously observed in elderly men (27) and middle-aged women (11). However, our results do not indicate a longitudinal association between D9D and CRP whereas a high D9D activity seemed to be predictive for elevated CRP in men followed for $20 \mathrm{y}$ (age 50-70 y) (13). One reason for the lack of a longitudinal association in our data might be that D9D (and also CRP) is mainly influenced by the current diet and other factors like the current weight status. Hence, D9D measured at the time of CRP assessment can be expected to have a stronger association than D9D measured 2 y before a CRP measurement. In addition, diet is likely to change rapidly during childhood such that baseline D9D plays a less relevant role as marker of risk of systemic inflammation after $2 \mathrm{y}$. Examples for nutrients that are known to affect D9D activity by modulating SCD1 gene expression are SFA, PUFA, and carbohydrates. High intakes of SFA and carbohydrates were shown to increase and dietary PUFA were shown to decrease D9D $(7,27)$. Accordingly, a study with step-wise 
Table 1. Characteristics of the cross-sectional and longitudinal analysis group by CRP level ${ }^{\mathrm{a}}$

\begin{tabular}{|c|c|c|c|c|}
\hline & \multicolumn{2}{|c|}{ Cross-sectional population $(N=1943)^{\mathrm{b}}$} & \multicolumn{2}{|c|}{ Longitudinal population $(N=900)^{c}$} \\
\hline & 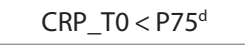 & CRP_T0 $\leq$ P75 ${ }^{\mathrm{d}}$ & 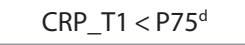 & CRP_T1 $1 \geq P 75^{\mathrm{d}}$ \\
\hline Number of children (\%) & $1223(62.9)$ & $720(37.1)$ & $553(61.4)$ & $347(38.6)$ \\
\hline Number of girls (\%) & $604(62.2)$ & $367(37.8)$ & $283(62.1)$ & $173(37.9)$ \\
\hline Mean age (SD) at T0, y & $6.14(1.76)$ & $6.55(1.73)$ & $6.20(1.71)$ & $6.76(1.55)$ \\
\hline \multicolumn{5}{|l|}{ Weight in categories at T0, $n$ (\%) ref. (25) } \\
\hline Thin & $93(79.5)$ & $24(20.5)$ & $44(84.6)$ & $8(15.4)$ \\
\hline Normal weight & $732(74.2)$ & $255(25.8)$ & $348(77.0)$ & $104(23.0)$ \\
\hline Overweight & $274(62.3)$ & $166(37.7)$ & $118(55.9)$ & $93(44.1)$ \\
\hline Level 3,4 & $698(62.5)$ & $419(37.5)$ & $311(60.4)$ & 204 (39.6) \\
\hline Level 5, 6 & $336(67.5)$ & $162(32.5)$ & $170(70.0)$ & $73(30.0)$ \\
\hline Desaturase activity at T0 & Mean $(99 \% \mathrm{Cl})$ & Mean $(99 \% \mathrm{Cl})$ & Mean $(99 \% \mathrm{Cl})$ & Mean $(99 \% \mathrm{Cl})$ \\
\hline D9D & $0.048(0.047 ; 0.049)$ & $0.053(0.052 ; 0.055)$ & $0.048(0.046 ; 0.049)$ & $0.053(0.050 ; 0.055)$ \\
\hline D6D & $0.068(0.067 ; 0.070)$ & $0.071(0.069 ; 0.072)$ & $0.068(0.066 ; 0.070)$ & $0.074(0.071 ; 0.076)$ \\
\hline D5D & $6.215(6.119 ; 6.312)$ & $6.231(6.093 ; 6.368)$ & $6.310(6.169 ; 6.450)$ & $6.061(5.876 ; 6.245)$ \\
\hline Palmitic acid, 16:0 (\%wt) & $25.8(25.7 ; 25.9)$ & $25.8(25.7 ; 26.0)$ & $25.7(25.5 ; 25.9)$ & $25.8(25.6 ; 26.0)$ \\
\hline Palmitoleic acid, 16:1n-7 (\%wt) & $1.25(1.22 ; 1.28)$ & $1.39(1.34 ; 1.43)$ & $1.23(1.19 ; 1.28)$ & $1.37(1.30 ; 1.44)$ \\
\hline Linoleic acid, 18:2n-6 (\%wt) & $18.2(18.1 ; 18.4)$ & $18.1(17.9 ; 18.3)$ & $18.2(18.0 ; 18.4)$ & $18.1(17.8 ; 18.4)$ \\
\hline CRP, girls (mg/l) & $0.30(0.60 ; 0.90)$ & $2.00(3.50 ; 6.90)$ & $0.40(0.90 ; 0.45)$ & $1.20(2.10 ; 5.10)$ \\
\hline CRP z-score & $-0.15(0.19 ; 0.59)$ & $1.31(1.75 ; 2.21)$ & $-0.11(0.56 ; 1.75)$ & $0.88(1.34 ; 2.12)$ \\
\hline \multicolumn{5}{|l|}{ CRP at T1 } \\
\hline CRP (mg/l) & & & $0.20(0.40 ; 0.70)$ & $1.60(2.90 ; 6.70)$ \\
\hline CRP, boys (mg/l) & & & $0.20(0.30 ; 0.50)$ & $1.60(2.80 ; 6.60)$ \\
\hline CRP, girls (mg/l) & & & $0.30(0.50 ; 0.70)$ & $1.80(3.00 ; 6.90)$ \\
\hline CRP z-score & & & $-0.05(0.22 ; 0.61)$ & $1.34(1.74 ; 2.33)$ \\
\hline $\begin{array}{l}\mathrm{Cl} \text {, confidence interval; } \text { CRP, C-reactive protein; } \\
\text { a } \mathrm{P} \text { roups were categorized according to the } 75 \text { th } \\
\text { were below or equal to the lower detection limi } \\
\text { of } 900 \text { children }(33.3 \%) \text { this was the case at T1. }{ }^{\circ} \\
\text { sectional population. } 755 \text { indicates the } 75 \text { th per }\end{array}$ & $\begin{array}{l}\text { ntile; ISCED, International } \\
\text { ntile of the age- and sex-s }\end{array}$ & $\begin{array}{l}\text { Classification of Education } \\
\text { PP reference values. }{ }^{\circ} \text { Cross } \\
\text { hildren ( } 31.0 \%) \text { CRP value } \\
\text { erence population (26). }{ }^{\text {e }} \\
\text { udinal population. }\end{array}$ & $\begin{array}{l}\text { ressed as weight percent } \\
\text { opulation: In } 599 \text { of } 1943 \\
\text { w or equal to the lower de } \\
\text { the } 75 \text { th percentile of the }\end{array}$ & $\begin{array}{l}\text { all FA detected (\%wt). } \\
n \text { ( } 30.8 \%) \text { CRP values } \\
n \text { limit at T0 and in } 300 \\
\text { sis group of our cross- }\end{array}$ \\
\hline
\end{tabular}

increases of carbohydrate intake confirmed that a high intake promotes an increase of blood palmitoleic acid (15) which could have been caused by increased D9D activity. Thus, dietary changes may have attenuated the relation with CRP. Due to the lack of FA data at follow-up, unfortunately changes in D9D over the 2 y period could not be considered in the present analysis.
Previous studies have shown an association between D9D activity and components of the metabolic syndrome $(9,10,23,28)$ and between increased CRP and the metabolic syndrome (29). If a decrease in D9D activity decreases CRP and thus subclinical inflammation, this may also result in beneficial effects on metabolic syndrome components. On the other hand, reverse causation cannot be ruled out, i.e., it would 


\section{Articles $\quad$ Wolters et al.}

Table 2. Odds ratios for having CRP $\geq 75^{\text {th }}$ age- and sex-specific percentile by 1 unit difference in estimated desaturase activity

\begin{tabular}{|c|c|c|c|c|c|c|}
\hline \multirow[b]{4}{*}{$\begin{array}{l}\text { Desaturase } \\
\text { activity at T0 }\end{array}$} & \multicolumn{2}{|c|}{$\begin{array}{l}\text { Cross-sectional } \\
\text { model }^{a}\end{array}$} & \multicolumn{4}{|c|}{ Longitudinal models $^{\mathrm{b}}$} \\
\hline & \multirow{2}{*}{\multicolumn{2}{|c|}{$\begin{array}{l}\text { Effect on T0 CRP, } \\
\qquad N=1943\end{array}$}} & \multicolumn{4}{|c|}{ Effect on $\mathrm{T} 1 \mathrm{CRP}, \mathrm{N}=900$} \\
\hline & & & \multicolumn{2}{|c|}{$\begin{array}{l}\text { Not adjusted for } \\
\text { T0 CRP z-score }\end{array}$} & \multicolumn{2}{|c|}{$\begin{array}{c}\text { Adjusted for T0 CRP } \\
\text { z-score }\end{array}$} \\
\hline & $\begin{array}{l}\text { Odds } \\
\text { ratio }\end{array}$ & $\begin{array}{c}99 \% \\
\mathrm{Cl}\end{array}$ & $\begin{array}{l}\text { Odds } \\
\text { ratio }\end{array}$ & $\begin{array}{c}99 \% \\
\mathrm{Cl}\end{array}$ & $\begin{array}{l}\text { Odds } \\
\text { ratio }\end{array}$ & $\begin{array}{c}99 \% \\
\mathrm{Cl}\end{array}$ \\
\hline $\mathrm{D}^{\mathrm{C}} \mathrm{D}^{\mathrm{c}}$ & 1.11 & $1.01 ; 1.22^{*}$ & 1.05 & $0.91 ; 1.21$ & 1.01 & $0.87 ; 1.18$ \\
\hline $\mathrm{D}^{1} \mathrm{D}^{\mathrm{c}}$ & 1.02 & $0.94 ; 1.11$ & 1.05 & $0.92 ; 1.20$ & 1.05 & $0.91 ; 1.21$ \\
\hline D5D & 1.05 & $0.95 ; 1.15$ & 0.94 & $0.81 ; 1.10$ & 0.93 & $0.79 ; 1.09$ \\
\hline
\end{tabular}

$\mathrm{Cl}$, confidence interval; CRP, C-reactive protein; ISCED, International Standard Classification of Education

"Models were adjusted for age, sex, country, maximum ISCED level of parents, and BMI $\mathrm{z}$-score. ${ }^{\mathrm{b}}$ The same adjustments were made as under 1 additionally including a binary variable for control vs. intervention regions. For statistical analysis, D9D and D6D were multiplied by 100 , i.e., the scale was converted to receive meaningful effect estimates in the models. * statistical significant

also be possible that CRP reduction decreases D9D activity. In adipocytes deficient in the D9D isoform, $S C D 1$, expression of the proinflammatory factors monocyte chemoattractant protein 1 (MCP-1) and interleukin (IL)-6 was significantly reduced compared with control adipocytes (30). This would lead to a reduction in CRP produced in response to inflammatory signals, particularly IL-6 (26).

In contrast to our results, D6D was positively and D5D was negatively associated with CRP in boys in a cross-sectional Japanese study with 237 school children aged $11.5 \pm 1.5 \mathrm{y}$. In the same study, no association was seen in girls, which may partly be due to sex hormone effects on D6D and D5D (24). Studies indicate that estrogen seems to stimulate and testosterone seems to inhibit the conversion of the precursor FA to arachidonic and eicosapentaenoic acid (31). However, such effects may be relevant in the Japanese study including school children with a mean age of around 11 y but are improbable in our sample of young and thus mostly prepubertal children. In 489 Japanese adults, serum CRP concentration was inversely associated with D5D in men and women (32).

Our results suggest that low D9D may be beneficial with view to subclinical inflammation If there was a causal relationship between D9D and CRP, dietary modifications such as a decrease of SFA or carbohydrate intake may result in lower D9D and CRP as D9D expression can be induced by these nutrients $(7,15)$, Furthermore, variations in the SCD1 gene have been shown to influence obesity and metabolic risk factors (33) and may thus affect CRP levels although no such association was seen in a sample of middle-aged adults (34).

In conclusion, our data show that cross-sectionally D9D is positively associated with CRP independent of the weight status in young children. High D9D may indicate an increased risk for subclinical inflammation in young children but may not predict subsequent inflammatory status.

\section{METHODS}

In this study, data of a subsample of the IDEFICS (Identification and prevention of dietary- and lifestyle-induced health effects in children

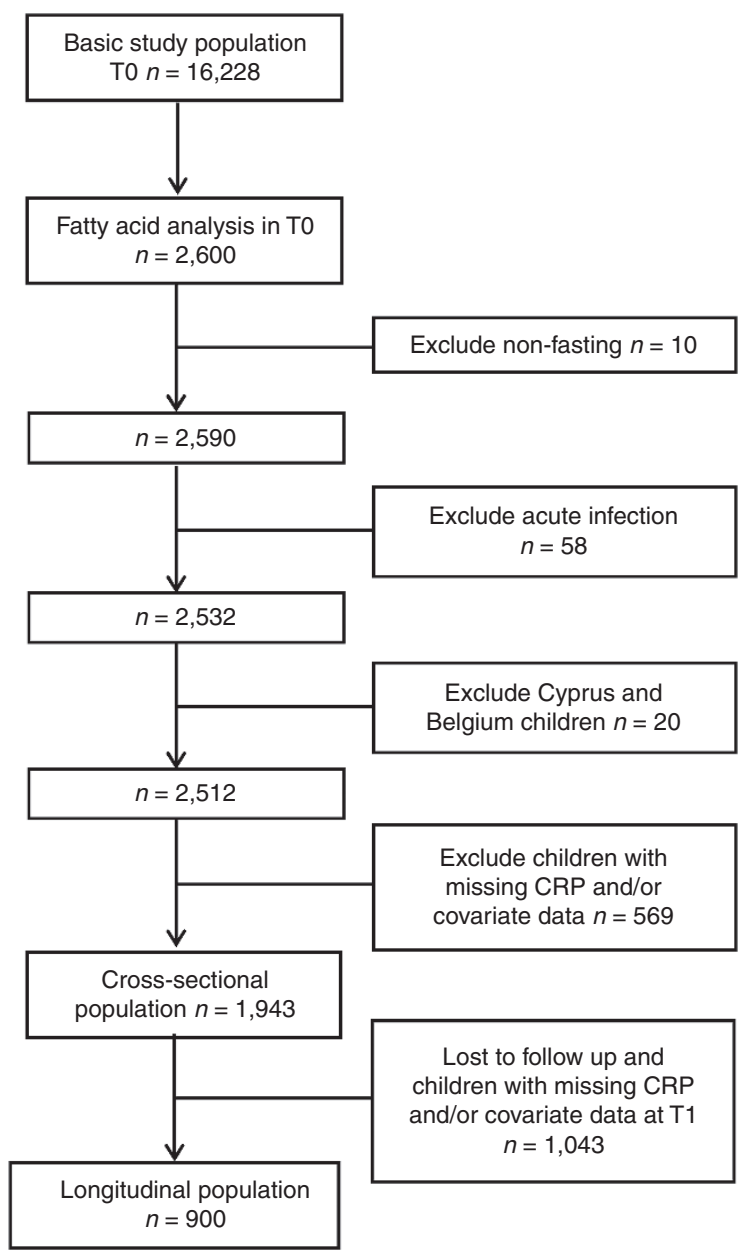

Figure 1. Flow chart of the inclusion and exclusion of IDEFICS participants for the cross-sectional and longitudinal analysis. IDEFICS, Identification and prevention of dietary- and lifestyle-induced health effects in children and infants.

and infants) cohort were analyzed. In the IDEFICS baseline survey (T0), 16,228 children aged 2 to $<10 \mathrm{y}$ from eight European countries were examined in 2007/2008. After 2 y (T1) 11,041 of these children were re-examined. The study design has been described in detail elsewhere (35).

\section{Ethics Statement}

The participating centers in each country obtained ethical approval by the competent Institutional Review Boards. For the six countries which provided data for the present analyses these were the following ethics committees: Ethics Committee of the University of Bremen, Bremen, Germany; Tallinn Medical Research Ethics Committee, Tallinn, Estonia; Azienda Sanitaria Locale Avellino Comitato Etico, Avellino, Italy; Regionala Etikprövningsnämnden I Göteborg, Gothenburg, Sweden; Comité Ético de Investigación Clínica de Aragón, Zaragoza, Spain; and Egészségügyi Tudományos Tanács, Pécs, Hungary.

The examination program included standard anthropometric measurements such as body weight and height, a personal interview on health conditions of the child, questionnaires on parental information like educational level and collection of blood samples, as described in detail elsewhere (35).

Fasting blood samples were obtained either by collecting a drop of blood from a fingertip or by venipuncture. A rapid gas-liquid chromatography method was applied to analyze FA composition of whole blood as previously described (36). FA are expressed as weight percentage of all FA detected (\%wt). The estimated desaturase activities 
were determined from the FA product-precursor-ratios as follows: D9D: 16:1n-7/16:0, D6D: 20:3n-6/18:2n-6, and D5D: 20:4n-6/20:3n-6. D9D and D6D were rescaled, i.e., multiplied by 100 to receive meaningful effect estimates. Body mass index was converted to age- and sex-specific z-scores using the reference values according to Cole and Lobstein (25).

For the assessment of high-sensitivity CRP concentrations in serum latex-enhanced nephelometry (BN2-Nephelometer, Siemens, Eschborn, Germany) was used. The serum CRP values were measured with a precision of $0.1 \mathrm{mg} / \mathrm{l}$ and a lower detection limit of $0.2 \mathrm{mg} / \mathrm{l}$.

The FA profiles from T0 fasting blood samples were analyzed in a subsample of 2,600 participants aged 2 to $<10$ y with oversampling of overweight and obese children. Exclusion criteria were blood drawn in a nonfasting state and acute infections/inflammation defined as CRP level $\geq 10 \mathrm{mg} / \mathrm{l}(26)$. As only few children from Cyprus $(N=2)$ and Belgium $(N=18)$ provided valid FA data, these two countries were not included in the analysis. Figure 1 shows selection of the analysis groups for the cross-sectional and longitudinal analyses. The cross-sectional analysis comprises only children with full information on FA, CRP, and covariates (age, sex, country, International Standard Classification of Education (ISCED) level, and BMI z-score) at baseline $(N=1,943)$. For inclusion in the longitudinal analysis, information on CRP at T1 was required in addition $(N=900$ children, due to dropout or missing CRP measurements).

Because of the left-truncated distribution of CRP (due to the large amount of values below the detection limit), CRP values were categorized in being above $(\geq)$ vs. below the 75th age- and sex-specific percentile based on recently published reference values (26) for young children. This binary variable was used in the later logistic models. Additionally, age- and sex-specific CRP z-scores were calculated based on the previously mentioned reference values.

As potential confounders, age (continuous), sex, BMI z-score, country and maximum ISCED level of parents were included as covariates. ISCED was aggregated to three categories; low level $=0,1,2$; medium $=3,4$; high $=5,6$. Additionally, a binary variable indicating control vs. intervention regions was used to adjust for potential differences resulting from an intervention implemented in the study (37).

Logistic regression models were used to assess the cross-sectional associations between T0 CRP (CRP $\geq$ P75 vs. CRP $<$ P75 (reference)) and the T0 exposures of interest (D9D, D6D or D5D) adjusting for age, sex, BMI z-score, country, and parents' maximum ISCED level. In the longitudinal analyses, again logistic regression models were used to assess the associations between the exposures D9D, D6D, and D5D measured at T0 and CRP category at T1 adjusting for the abovementioned covariates, adding also an indicator for control vs. intervention region. As children with a baseline CRP value close to the 75th percentile may be more likely to exceed the 75th percentile at T1 compared with children with lower baseline CRP, the longitudinal model was in a second step additionally adjusted for the baseline CRP z-scores. All analyses were performed using SAS statistical software version 9.3 (SAS Institute, Cary, NC). To account at least partially for multiple testing we used the $99 \%$ CI to determine statistical significance.

\section{STATEMENT OF FINANCIAL SUPPORT}

We gratefully acknowledge the financial support of the European Community within the Sixth RDT Framework Programme Contract no. 016181 (Food).

Disclosures and conflict of interest: None.

\section{REFERENCES}

1. Choi J, Joseph L, Pilote L. Obesity and C-reactive protein in various populations: a systematic review and meta-analysis. Obes Rev 2013;14:232-44.

2. Ahrens W, Pigeot I, Pohlabeln H, et al.; IDEFICS consortium. Prevalence of overweight and obesity in European children below the age of 10. Int J Obes (Lond) 2014;38 (Suppl 2):S99-107.

3. Lobstein T, Jackson-Leach R, Moodie ML, et al. Child and adolescent obesity: part of a bigger picture. Lancet 2015;385:2510-20.

4. Santos S, Oliveira A, Lopes C. Systematic review of saturated fatty acids on inflammation and circulating levels of adipokines. Nutr Res 2013;33:687-95.
5. Calder PC. Marine omega- 3 fatty acids and inflammatory processes: Effects, mechanisms and clinical relevance. Biochim Biophys Acta 2015;1851:469-84.

6. Liu X, Strable MS, Ntambi JM. Stearoyl CoA desaturase 1: role in cellular inflammation and stress. Adv Nutr 2011;2:15-22.

7. Sampath H, Ntambi JM. The role of stearoyl-CoA desaturase in obesity, insulin resistance, and inflammation. Ann NY Acad Sci 2011;1243:47-53.

8. Paton CM, Ntambi JM. Biochemical and physiological function of stearoyl-CoA desaturase. Am J Physiol Endocrinol Metab 2009;297:E28-37.

9. Chow LS, Li S, Eberly LE, et al. Estimated plasma stearoyl co-A desaturase-1 activity and risk of incident diabetes: the Atherosclerosis Risk in Communities (ARIC) study. Metabolism 2013;62:100-8.

10. Wolters M, Schlenz H, Börnhorst C, et al.; IDEFICS Consortium. Desaturase activity is associated with weight status and metabolic risk markers in young children. J Clin Endocrinol Metab 2015;100:3760-9.

11. Jacobs S, Schiller K, Jansen EH, Boeing H, Schulze MB, Kröger J. Evaluation of various biomarkers as potential mediators of the association between $\Delta 5$ desaturase, $\Delta 6$ desaturase, and stearoyl-CoA desaturase activity and incident type 2 diabetes in the European Prospective Investigation into Cancer and Nutrition-Potsdam Study. Am J Clin Nutr 2015;102:155-64.

12. Cho JS, Baek SH, Kim JY, Lee JH, Kim OY. Serum phospholipid monounsaturated fatty acid composition and $\Delta$-9-desaturase activity are associated with early alteration of fasting glycemic status. Nutr Res 2014;34:733-41.

13. Petersson H, Basu S, Cederholm T, Risérus U. Serum fatty acid composition and indices of stearoyl-CoA desaturase activity are associated with systemic inflammation: longitudinal analyses in middle-aged men. Br J Nutr 2008;99:1186-9.

14. Rondanelli M, Klersy C, Perna S, et al. Effects of two-months balanced diet in metabolically healthy obesity: lipid correlations with gender and BMIrelated differences. Lipids Health Dis 2015;14:139.

15. Volk BM, Kunces LJ, Freidenreich DJ, et al. Effects of step-wise increases in dietary carbohydrate on circulating saturated Fatty acids and palmitoleic acid in adults with metabolic syndrome. PLoS One 2014;9:e113605.

16. Takkunen MJ, de Mello VD, Schwab US, Ågren JJ, Kuusisto J, Uusitupa MI. Associations of erythrocyte membrane fatty acids with the concentrations of C-reactive protein, interleukin 1 receptor antagonist and adiponectin in 1373 men. Prostaglandins Leukot Essent Fatty Acids 2014;91:169-74.

17. Bernstein AM, Roizen MF, Martinez L. Purified palmitoleic acid for the reduction of high-sensitivity C-reactive protein and serum lipids: a double-blinded, randomized, placebo controlled study. J Clin Lipidol 2014;8: 612-7.

18. Nestel P, Clifton P, Noakes M. Effects of increasing dietary palmitoleic acid compared with palmitic and oleic acids on plasma lipids of hypercholesterolemic men. J Lipid Res 1994;35:656-62.

19. Ratnayake WM, Galli C. Fat and fatty acid terminology, methods of analysis and fat digestion and metabolism: a background review paper. Ann Nutr Metab 2009;55:8-43.

20. Giudetti AM, Cagnazzo R. Beneficial effects of n-3 PUFA on chronic airway inflammatory diseases. Prostaglandins Other Lipid Mediat 2012;99:57-67.

21. Merino DM, Ma DW, Mutch DM. Genetic variation in lipid desaturases and its impact on the development of human disease. Lipids Health Dis 2010;9:63.

22. Martinelli N, Girelli D, Malerba G, et al. FADS genotypes and desaturase activity estimated by the ratio of arachidonic acid to linoleic acid are associated with inflammation and coronary artery disease. Am J Clin Nutr 2008;88:941-9.

23. Do HJ, Chung HK, Moon J, Shin MJ. Relationship between the estimates of desaturase activities and cardiometabolic phenotypes in Koreans. J Clin Biochem Nutr 2011;49:131-5.

24. Saito E, Okada T, Abe Y, et al. Abdominal adiposity is associated with fatty acid desaturase activity in boys: implications for C-reactive protein and insulin resistance. Prostaglandins Leukot Essent Fatty Acids 2013;88: 307-11.

25. Cole TJ, Lobstein T. Extended international (IOTF) body mass index cutoffs for thinness, overweight and obesity. Pediatr Obes 2012;7:284-94.

26. Schlenz H, Intemann T, Wolters M, et al.; IDEFICS consortium. C-reactive protein reference percentiles among pre-adolescent children in Europe based on the IDEFICS study population. Int J Obes (Lond) 2014;38 Suppl 2:S26-31. 


\section{Articles | Wolters et al.}

27. Petersson H, Lind L, Hulthe J, Elmgren A, Cederholm T, Risérus U. Relationships between serum fatty acid composition and multiple markers of inflammation and endothelial function in an elderly population. Atherosclerosis 2009;203:298-303.

28. Jacobs S, Schiller K, Jansen E, et al. Association between erythrocyte membrane fatty acids and biomarkers of dyslipidemia in the EPIC-Potsdam study. Eur J Clin Nutr 2014;68:517-25.

29. Wärnberg J, Marcos A. Low-grade inflammation and the metabolic syndrome in children and adolescents. Curr Opin Lipidol 2008;19:11-5.

30. Liu X, Miyazaki M, Flowers MT, et al. Loss of Stearoyl-CoA desaturase-1 attenuates adipocyte inflammation: effects of adipocyte-derived oleate. Arterioscler Thromb Vasc Biol 2010;30:31-8.

31. Decsi T, Kennedy K. Sex-specific differences in essential fatty acid metabolism. Am J Clin Nutr 2011;94(6 Suppl):1914S-9S.

32. Poudel-Tandukar K, Sato M, Ejima Y, et al. Relationship of serum fatty acid composition and desaturase activity to C-reactive protein in Japanese men and women. Atherosclerosis 2012;220:520-4.
33. Warensjö E, Ingelsson E, Lundmark P, et al. Polymorphisms in the SCD1 gene: associations with body fat distribution and insulin sensitivity. Obesity (Silver Spring) 2007;15:1732-40.

34. Arregui M, Buijsse B, Stefan N, et al. Heterogeneity of the Stearoyl-CoA desaturase-1 (SCD1) gene and metabolic risk factors in the EPIC-Potsdam study. PLoS One 2012;7:e48338.

35. Ahrens W, Bammann K, Siani A, et al.; IDEFICS Consortium. The IDEFICS cohort: design, characteristics and participation in the baseline survey. Int J Obes (Lond) 2011;35 (Suppl 1):S3-15.

36. Marangoni F, Colombo C, Galli C. A method for the direct evaluation of the fatty acid status in a drop of blood from a fingertip in humans: applicability to nutritional and epidemiological studies. Anal Biochem 2004;326:267-72.

37. De Henauw S, Verbestel V, Mårild S, et al.; IDEFICS Consortium. The IDEFICS community-oriented intervention programme: a new model for childhood obesity prevention in Europe? Int J Obes (Lond) 2011;35 Suppl 1:S16-23. 